\title{
Evidence of a Transition from Nonlinear to Linear Screening of a Two-Dimensional Electron System Detected by Photoluminescence Spectroscopy
}

\author{
M. Yamaguchi, ${ }^{1,2}$ S. Nomura, ${ }^{1,2,3}$ T. Maruyama, ${ }^{4}$ S. Miyashita, ${ }^{4}$ Y. Hirayama, ${ }^{5}$ H. Tamura,,${ }^{1,2}$ and T. Akazaki ${ }^{1,2}$ \\ ${ }^{1}$ NTT Basic Research Laboratories, NTT Corporation, 3-1 Morinosato-Wakamiya, Atsugi-shi, Kanagawa 243-0198, Japan \\ ${ }^{2}$ CREST, Japan Science and Technology Agency, Honcho, Kawaguchi, Saitama 332-0012, Japan \\ ${ }^{3}$ Institute of Physics, University of Tsukuba, 1-1-1 Tennodai Tsukuba, Ibaraki 305-8571, Japan \\ ${ }^{4}$ NTT Advanced Technology, 3-1 Morinosato-Wakamiya, Atsugi-shi, Kanagawa 243-0198, Japan \\ ${ }^{5}$ Department of Physics, Tohoku University, Aoba-ku, Sendai, Miyagi 980-8578, Japan
}

(Received 6 February 2008; published 11 November 2008)

\begin{abstract}
We clearly identify single-electron-localization (SEL), nonlinear screening (NLS), and linear screening (LS) regimes of gate induced electrons in a GaAs quantum well from photoluminescence spectra and intergate capacitance. Neutral and charged excitons observed in the SEL regime rapidly lose their oscillator strength when electron puddles are formed, which mark the onset of NLS. A further increase in the density of the electrons induces the transition from the NLS to LS, where the emission of a charged exciton changes to the recombination of two-dimensional electron gas and a hole.
\end{abstract}

DOI: 10.1103/PhysRevLett.101.207401

PACS numbers: 78.67.De, 73.20.Jc, 78.55.Cr

When a two-dimensional electron system (2DES) is formed at a semiconductor interface with a disorder potential, the screening of the potential fluctuation by the accumulated electrons plays an essential role in keeping the electron density homogeneous. At a high electron density the screening greatly reduces the random potential by $1 / 2 q_{0} s \ll 1$, where $q_{0}$ is the inverse screening length in the linear screening (LS) regime and $s$ is the distance from the disorder source [1]. With decreasing electron density, electrons begin to be depleted around the disorder potential "hilltops" and spatially inhomogeneous states emerge [2]. It has been experimentally shown [3] that a disorder-driven percolation transition is a generic mechanism for the metal-insulator transition (MIT) [4,5] in an undoped $\mathrm{GaAs} / \mathrm{AlGaAs}$ heterojunction due to the breakdown of the screening in the random potential. Resistivity and compressibility measurements [6] have shown that the percolation transition occurs at a much lower density than that at which the crossover does in the sign of $\partial R / \partial T$ and that it can be described by nonlinear screening (NLS) theory $[1,7,8]$.

In transport measurement, the percolation transition is discussed by fitting the conductivity and obtaining the critical exponent characterizing the vanishing of the conductivity [3]. Optical measurement is more suitable for a direct observation of the dilute 2DES in the insulating regime [9-12]. A neutral exciton $X^{0}$ and a negatively charged exciton $X^{-}$have been observed in a low-density regime $[10,13,14]$. This suggests that $X^{-}$is formed as a result of a photoexcited electron-hole pair binding to a localized electron in the random potential of remote ionized donors. In this dilute 2DES regime, the nature of the random potential plays an important role. The length scale of potential fluctuations in a quantum well $(\mathrm{QW})$ is determined by the distance $s$ between the ionized impurity layer and the QW. In a modulation-doped sample, the distance $s$ is short (typically $15-50 \mathrm{~nm}$ ) and, as the density increases, electrons first occupy different potential valleys to avoid an increase in Coulomb energy and then transit to the dilute 2DES. On the other hand, in an undoped GaAs sample, the dominant potential fluctuation may originate not from the random potential of the remote ionized donor layer but from the random distribution of surface pinning positions. In this case the distance $s$ can be larger than a few hundred nanometers, which is much larger than that in a modulation-doped GaAs QW. It is very important to study the properties of the dilute 2DES in an undoped QW with such a long-range random fluctuation.

In this Letter, on the basis of the results of lowtemperature $(100 \mathrm{mK})$ intergate capacitance and photoluminescence (PL) measurements, we study the transition of electron states in an undoped GaAs QW with changing electron density. From the intergate capacitance, which directly reflects the electron screening length $d_{w}$ [15], we deduce two characteristic voltages: one at which electrons begin to accumulate in the $\mathrm{QW}$, and the other at which $d_{w}$ has the minimum. These characteristic voltages consistently match the change in the bias voltage dependence of the PL intensity and the linewidth of charged excitons, which clearly reveal three different electron states: a single-electron-localization (SEL) regime, an NLS regime, and an LS regime.

The sample was an $S=1 \times 1 \mathrm{~mm}^{2} 20$-nm GaAs QW sandwiched by undoped $\mathrm{Al}_{0.2} \mathrm{Ga}_{0.8} \mathrm{As}$ barriers grown by molecular beam epitaxy, as shown in Fig. 1(b). The background impurity was $p$-type and below $1 \times 10^{14} \mathrm{~cm}^{-3}$. A semitransparent $\mathrm{Ti} / \mathrm{Au}$ film deposited on the surface and a heavily $n$-doped GaAs wafer worked as front and back gates, respectively, which were separated by $d_{1}=250 \mathrm{~nm}$ and $d_{2}=620 \mathrm{~nm}$ from the QW, respectively. AuGeNi was 
(a)

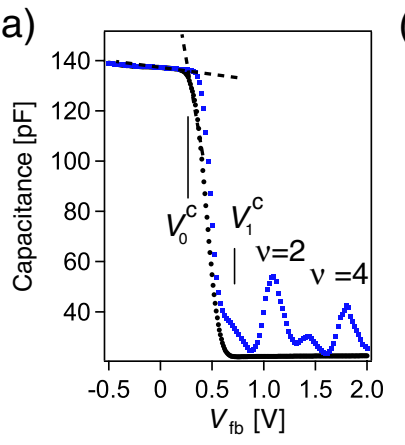

(b)

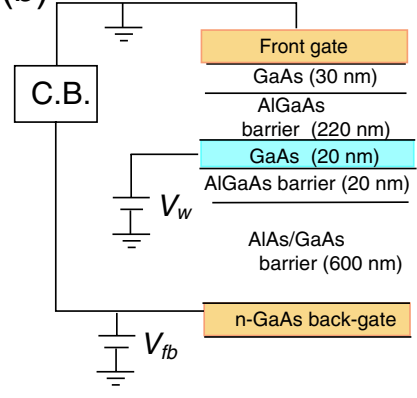

(c)

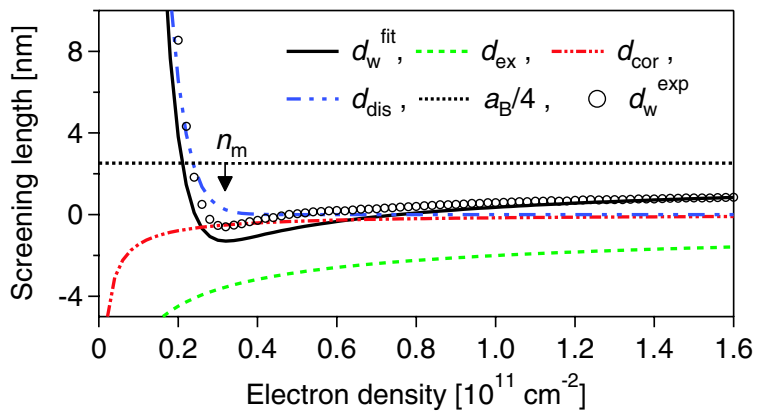

FIG. 1 (color online). (a) The capacitance spectra between the gates at $0 \mathrm{~T}$ (black circles) and $1.5 \mathrm{~T}$ (blue or dark gray squares). (b) The layer structure of the sample and a diagram of the intercapacitance measurement using a capacitance bridge (C.B.). (c) The screening length $d_{w}^{\text {exp }}$, plotted as a function of the estimated electron density (open circles). The solid curve is the fit to the nonlinear screening theory $d_{w}^{\exp }=a_{B} / 4+\Delta d_{\text {dis }}+$ $\Delta d_{\text {ex }}+\Delta d_{\text {cor }}$. (See text for details.)

alloyed to provide an Ohmic contact to the QW. The Ohmic contact was kept at $V_{w}=-0.3 \mathrm{~V}$, and electrons were supplied from this contact when the gate bias $V_{\mathrm{fb}}$ was applied. The laser light was introduced to the sample surface through an optical fiber with an excitation power density $<1 \mathrm{~mW} / \mathrm{cm}^{2}$ at $800 \mathrm{~nm}$. The PL was collected with the same fiber and detected with a 1-m monochromator equipped with a liquid nitrogen-cooled charge-coupled device. The intergate capacitance between the front and back gates was measured with a capacitance-bridge circuit at $0.12-1 \mathrm{kHz}$ and at $30-\mathrm{mV}$ excitation.

By doing algebra similar to that in [15], we obtain the relation between the screening length $d_{w}=\left(\epsilon / e^{2}\right) \times$ $\left(\partial \mu / \partial n_{s}\right)$ and intergate capacitance $C^{-1}=$ $\left(d_{1} d_{2} / \epsilon S d_{w}\right)+C_{\mathrm{fb}}^{-1}$, where $\mu$ and $n_{s}$ are the chemical potential and the electron density in the QW, $S \sim 1 \mathrm{~mm}^{2}$ is the sample area, and $C_{\mathrm{fb}}=\epsilon S /\left(d_{1}+d_{2}\right)$ is the geometric capacitance between the gates at $n_{s}=0$. The NLS theory predicts that $d_{w}\left(n_{s}\right)$ is represented by the sum of the contribution of the single-particle density of states $a_{B} / 4$ ( $a_{B}$ : the effective Bohr radius), exchange correction $\Delta d_{\text {ex }}\left(n_{s}\right)$, correlation effect $\Delta d_{\text {cor }}\left(n_{s}\right)$, and disorder potential $\Delta d_{\text {dis }}\left(n_{s}\right)[6]$.

The $V_{\mathrm{fb}}$ dependence of intergate capacitance for $B=$ $0 \mathrm{~T}$ and $1.5 \mathrm{~T}$ is plotted in Fig. 1(a). The capacitance, which is almost constant at low $V_{\mathrm{fb}}$, suddenly drops around $V_{\mathrm{fb}}=V_{0}^{\mathrm{C}}=0.3 \mathrm{~V}$, where the electrons start to accumulate. An asymmetric minimum appears at $V_{\mathrm{fb}}=V_{1}^{\mathrm{C}}=$ $0.72 \mathrm{~V}$, which corresponds to the minimum value of $d_{w}$ shown in Fig. 1(c). The solid curve is the theoretical fit to the NLS theory; here, the $n_{i}\left(=2.0 \times 10^{12} \mathrm{~cm}^{-2}\right)$ is only the fitting parameter used to adjust the position of the minimum. The offset constant $d_{w}$ could not be accurately determined because of a stray capacitance of $\sim 15 \mathrm{pF}$. The experimental data for $d_{w}^{\text {exp }}$ in Fig. 1(c) are plotted so that they coincide with the theoretical value of $d_{w}^{\text {fit }}$ at $n_{s}=$ $1.6 \times 10^{11} \mathrm{~cm}^{-2}$. At $1.5 \mathrm{~T}$, two distinct peaks appear around $V_{\mathrm{fb}}=1.1$ and $1.8 \mathrm{~V}$, which originate from the incompressive quantum Hall states of $\nu=2$ and 4 [15]. The electron density $n_{s}$ can be estimated as $n_{s}=1.0 \times$ $10^{11}\left(V_{\mathrm{fb}}-V_{0}^{p}\right) \mathrm{cm}^{-2}$, where $V_{0}^{p} \simeq 0.4 \mathrm{~V}$, which is slightly larger than $V_{0}^{\mathrm{C}}=0.3 \mathrm{~V}$, at which the capacitance starts to drop. This discrepancy is discussed in the next paragraph.

Here, let us try to understand how electrons are accumulated in a QW with increasing $V_{\mathrm{fb}}$ in terms of the picture of percolation transition. In the very-low-density regime below $V_{\mathrm{fb}}<V_{0}^{\mathrm{C}}$ (regime I), individual potential valleys in the QW are empty or occupied by a single electron, and the measured capacitance $C$ is just the bare value, $C_{\mathrm{fb}}$. In this regime, the residual electrons, whose density is estimated to be $<1 \times 10^{9} \mathrm{~cm}^{-2}$, flow from the surface through the barrier. As the bias voltage increases as $V_{0}^{\mathrm{C}}<V_{f b}<V_{1}^{\mathrm{C}}$ (regime II), potential valleys are filled by degenerate electrons and puddlelike regions are created because the screening length, although consistently reduced by the electron density increase, is still sufficiently large to prevent the formation of a $2 \mathrm{DEG}$ extending over the entire QW region. This regime corresponds to the NLS regime. If there is no potential fluctuation in the QW and the manybody contribution is neglected, then $V_{0}^{p} \simeq 0.4 \mathrm{~V}$, extrapolated from the Landau peaks, should exactly correspond to the zero-density threshold. However, in the presence of potential fluctuation, it is reasonable to consider that $V_{0}^{p}$ corresponds to the threshold voltage for a percolation transition at which the half height of the potential valleys is filled by electrons. And the electron puddles are connected with each other to open a conduction path from end to end in the sample. Below $V_{0}^{p}$, because of the small compressibility $\partial n_{s} / \partial \mu$, which is proportional to $1 / d_{w}$, the increasing rate of $d n_{s} / d V_{\mathrm{fb}}$ becomes small and this may be why the voltage for the onset of electron accumulation $V_{\mathrm{fb}}=V_{0}^{\mathrm{C}} \simeq 0.3 \mathrm{~V}$ is even lower than $V_{0}^{p}$. From this consideration, we can estimate the amplitude of the potential fluctuation without screening by electrons as $\left(V_{0}^{p}-\right.$ $\left.V_{0}^{\mathrm{C}}\right) d_{2} /\left(d_{1}+d_{2}\right) \sim 30 \mathrm{meV}$. This is consistent with Ref. [1]'s $\sqrt{2 \pi} e^{2} \sqrt{n_{i}} / \epsilon=45 \mathrm{meV}$. With a further increase in the bias voltage $V_{\mathrm{fb}}>V_{1}^{\mathrm{C}}$ (regime III), the depletion regions (voids) disappear and the degenerate-electron gas finally covers the entire 2D plane, which corresponds to the LS regime. 
To investigate the above transition, we performed PL measurements. Figure 2(a) shows typical PL spectra measured in the three regimes described above and Fig. 2(b) shows color plots of the spectral evolution. In regime I, PL emissions for $X^{0}$ and $X^{-}$are observed. $X^{-}$is created by combining a photoexcited electron-hole pair and a single localized electron at the bottom of the disorder potential. In the middle of regime II at around $V_{\mathrm{fb}}=0.5 \mathrm{~V}$, the $X^{0}$ signal disappears and a single broad peak remains. In this regime, electron puddles are formed, and the accumulated electrons in the puddles weaken the binding energy between the electron and hole of a neutral and charged exciton. The long tail observed in Fig. 2(a) (indicated by arrows) is presumably due to the localization effect of the electrons and holes [16]. On the other hand, in regime III, where the electron density is higher than $3 \times 10^{10} \mathrm{~cm}^{-2}$, the line shape becomes even wider and more asymmetric. This is attributed to the formation of a 2DES extending over the entire region where the 2DES-hole recombination emission is dominant. In this regime, the localizationinduced tail disappears, which suggests that the potential fluctuation is reduced by screening. As we have seen in Fig. 2(b), the $X^{-}$peak transits smoothly to the 2DES-hole peak as $n_{s}$ is increased, and we hereafter tentatively name this series of peaks the $Y$ band.

The peak intensity and the linewidth obtained by fitting to an asymmetric Lorentzian curve are plotted in Fig. 3. Surprisingly, this result clearly shows the existence of the three distinct regimes, which is consistent with the result of the capacitance measurement depicted by the vertical lines at $V_{0}^{\mathrm{C}}$ and $V_{1}^{\mathrm{C}}$. In regime $\mathrm{I}$, the increase in the $X^{-}$intensity reflects the increase of the number of single localized electrons. The unchanged linewidth indicates that the $X^{-}$ in this regime does not interact with the third electron.
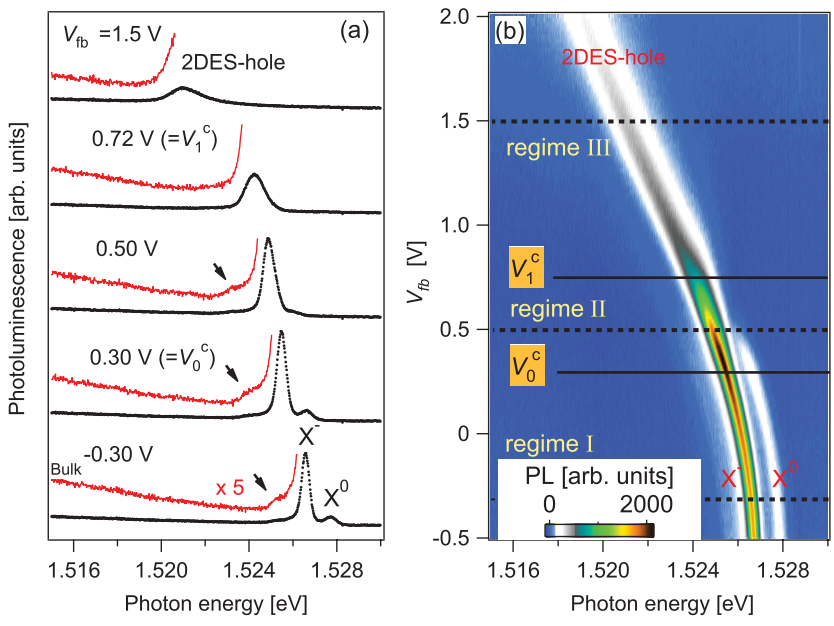

FIG. 2 (color). Typical PL spectra (a) in plots for different biases $\left(V_{\mathrm{fb}}\right)$ and $(\mathrm{b})$ in a color plot at $0 \mathrm{~T}$. The solid lines represent critical voltages $V_{0}^{\mathrm{C}}$ and $V_{1}^{\mathrm{C}}$ deduced from the capacitance measurement in Fig. 1 . The $V_{\mathrm{fb}}$-independent backgrounds are subtracted in the color plot.
There is a sharp transition at $V_{0}^{\mathrm{PL}} \sim 0.36 \mathrm{~V}$ where both the $X^{-}$and $X^{0}$ intensities suddenly decrease. As mentioned before, this is caused by the screening of the Coulomb interaction between the electron and hole by degenerateelectron puddles. The first PL transition at $V_{0}^{\mathrm{PL}}$ is consistently located between $V_{0}^{\mathrm{C}}$ and $V_{0}^{p}$, where degenerate electrons begin to accumulate, as we have argued previously. We can also see the increase in the linewidth with $n_{s}$. With the free $X^{-}$, which is moving in the $\mathrm{QW}$, the increase in the linewidth due to the $\left(X^{-}, e\right)$ scattering is discussed in [17]. We should note, however, that $X^{-}$observed in regime II is probably the localized one since the line shape of $X^{-}$in regime II is rather symmetric, while the free $X^{-}$has an asymmetric line shape due to the energy distribution of the recoil electron after the $X^{-}$recombination [18]. The second PL transition occurs over a wider range of $V_{1}^{\mathrm{PL}}$ of $0.75-$ $0.95 \mathrm{~V}$, where the slopes of both the intensity and linewidth change. This bias range is consistent with but a little higher than $V_{1}^{\mathrm{C}}=0.72 \mathrm{~V}$ determined in the capacitance measurement. According to the variational calculation for a percolation transition [8], the voids remaining at a density of $n_{m}$ at $V_{1}^{\mathrm{C}}=0.72 \mathrm{~V}$ completely disappear at a density of $(0.5 / 0.38) n_{m}$ corresponding to $V_{\mathrm{fb}} \sim 0.8 \mathrm{~V}$, which is in good agreement with the transition range. Therefore, the transition of the PL at $V_{1}^{\mathrm{PL}}$ surely corresponds to the transition from the NLS to LS regime.

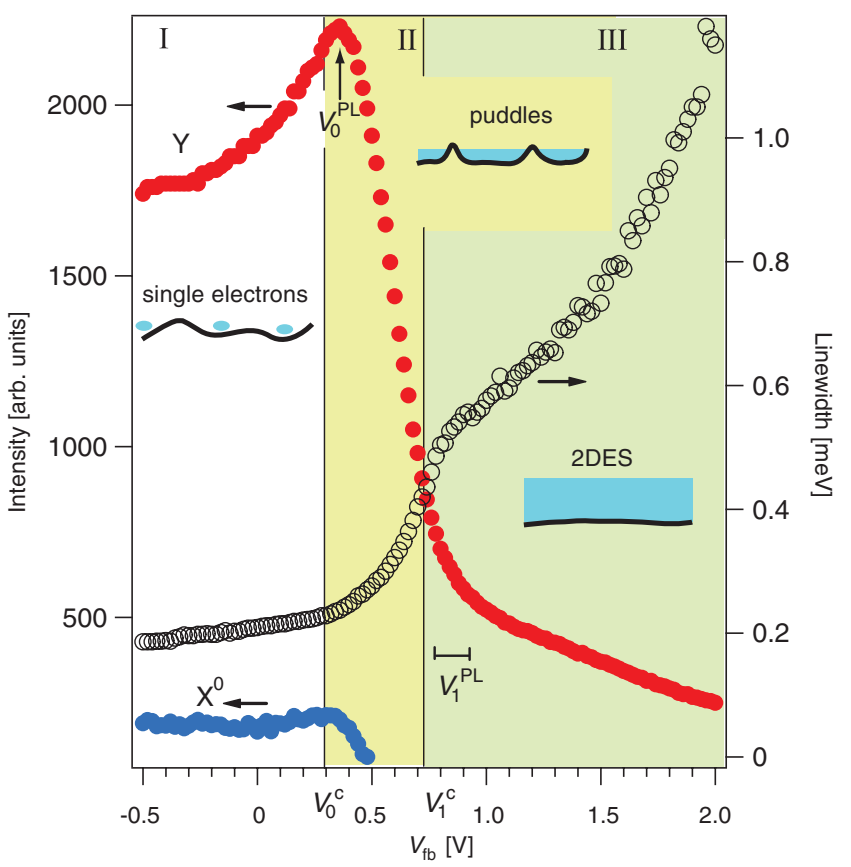

FIG. 3 (color). The PL peak intensity of the $Y$ band (i.e., $X^{-}$or 2DES-hole recombination peak) (red circles) and neutral exciton $\left(X^{0}\right)$ (blue circles) depending on $V_{\mathrm{fb}}$. Open circles represent the linewidth of the $Y$ band. The electron states of each regime (I, II, III), determined by the capacitance measurement, are shown schematically. 


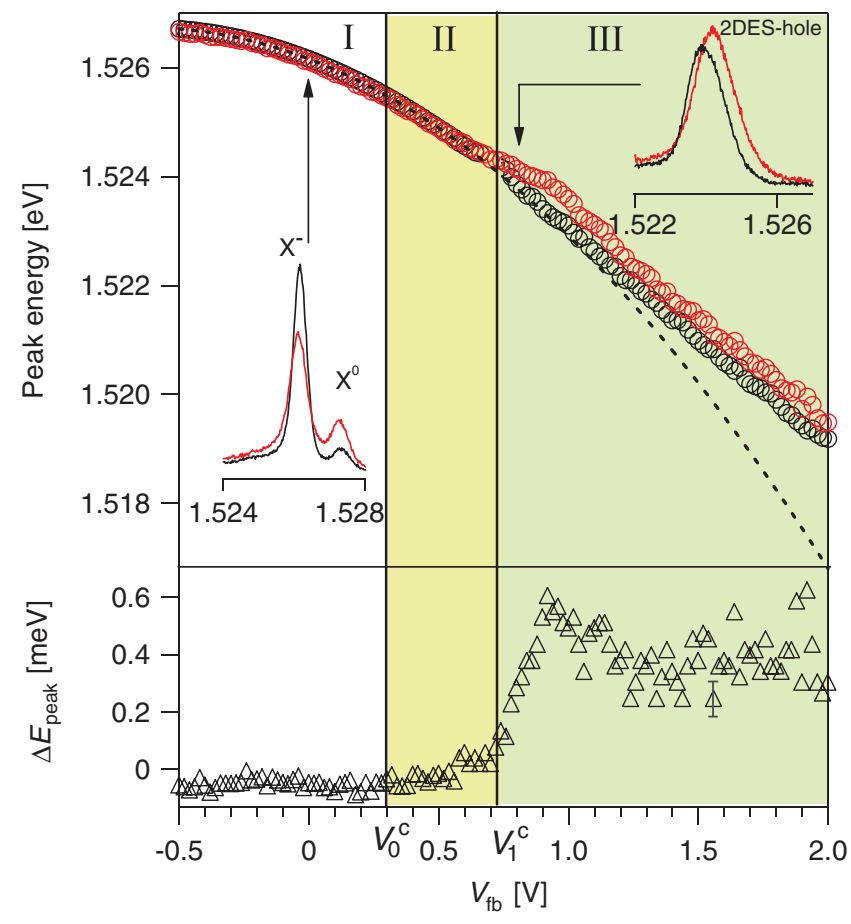

FIG. 4 (color). The emission energies for the $Y$ band at $100 \mathrm{mK}$ (black circles) and $4 \mathrm{~K}$ (red circles) are plotted in the upper panel. The dotted curve is the fit to a quadratic function of $V_{\mathrm{fb}}$ at $V_{\mathrm{fb}}<V_{0}^{\mathrm{C}}$ and is extrapolated to the higher $V_{\mathrm{fb}}$ regime. The insets are the PL spectra at $100 \mathrm{mK}$ (black line) and $4 \mathrm{~K}$ (red line). The difference in the peak energy between $4 \mathrm{~K}$ and $100 \mathrm{mK}$ is plotted in the lower panel.

We have found further clear evidence for the transition from the NLS regime to the LS regime. The peak energies of the $Y$ band at both $100 \mathrm{mK}$ and $4 \mathrm{~K}$, which are plotted in the upper panel of Fig. 4, deviate only above $V_{1}^{\mathrm{C}}$. We believe that the energy deviation arises from the thermal excitation of the weakly localized holes at a weakly fluctuating potential. As shown in the lower panel of Fig. 4, the energy difference of the peaks between $100 \mathrm{mK}$ and $4 \mathrm{~K}$ is $0.4 \mathrm{meV}$, which is comparable to the thermal energy of $4 \mathrm{~K}$. In regime III, the original potential fluctuation of about $30 \mathrm{meV}$ in regime I is screened and significantly reduced by the factor $1 / 2 q_{0} s \sim 1 / 100$ for $s=250 \mathrm{~nm}$, which results in a potential fluctuation amplitude below $0.3 \mathrm{meV}$.

To the best of our knowledge, most previous optical experiments have found only one kind of transition near the MIT $[10,11,19]$. As we have pointed out previously, this may be related to differences in the sample structure. For the electron puddle state to appear, the potential amplitude as well as the length scale of the potential fluctuation should be large. With a modulation-doped sample, the localized-electron state transits directly to the dilute 2DES without forming puddles, because the short range potential fluctuation cannot contain multiple electrons. In our undoped GaAs sample, $s \sim 250 \mathrm{~nm}$ (corresponding potential valley density $\sim 1.6 \times 10^{9} \mathrm{~cm}^{-2}$ ) is sufficiently large for the formation of electron puddles [20]. In addition, it is known that metal gate deposition causes interface roughness [21], which enhances the random potential amplitude. Electrical transport measurements in undoped samples have revealed only one kind of transition, i.e., the percolation transition [3], because it cannot cover the nonconducting regime.

In summary, we have clearly identified for the first time two transitions between three different electron regimes, i.e., the SEL regime, NLS regime, and LS regime by measuring both PL and capacitance. The result will provide an important contribution to our understanding of the microscopic mechanisms of the metal-insulator transition in the presence of the electronic screening effect in two dimensions.

[1] A. L. Efros, Solid State Commun. 70, 253 (1989).

[2] S. Ilani et al., Phys. Rev. Lett. 84, 3133 (2000); S. Ilani et al., Science 292, 1354 (2001).

[3] S. Das Sarma et al., Phys. Rev. Lett. 94, 136401 (2005).

[4] A. A. Shashkin et al., Phys. Rev. Lett. 73, 3141 (1994).

[5] S. V. Kravchenko and M. P. Sarachik, Rep. Prog. Phys. 67, 1 (2004); S. Das Sarma and E. H. Hwang, Solid State Commun. 135, 579 (2005).

[6] G. Allison et al., Phys. Rev. Lett. 96, 216407 (2006).

[7] A. L. Efros, F. G. Pikus, and V. G. Burnett, Phys. Rev. B 47, 2233 (1993).

[8] M. M. Fogler, Phys. Rev. B 69, 121409(R) (2004).

[9] P. Hawrylak, Phys. Rev. B 44, 3821 (1991).

[10] G. Finkelstein, H. Shtrikman, and I. Bar-Joseph, Phys. Rev. Lett. 74, 976 (1995).

[11] I. V. Kukushkin et al., Phys. Rev. B 53, R13 260 (1996).

[12] R. Kaur et al., Phys. Status Solidi A 178, 465 (2000).

[13] K. Kheng et al., Phys. Rev. Lett. 71, 1752 (1993).

[14] A. J. Shields et al., Phys. Rev. B 51, R18 049 (1995).

[15] J. P. Eisenstein, L. N. Pfeiffer, and K. W. West, Phys. Rev. B 50, 1760 (1994).

[16] G. Finkelstein, H. Shtrikman, and I. Bar-Joseph, Phys. Rev. B 53, 12593 (1996).

[17] A. Manassen et al., Phys. Rev. B 54, 10609 (1996).

[18] A. Esser et al., Phys. Rev. B 62, 8232 (2000).

[19] G. Yusa, H. Shtrikman, and I. Bar-Joseph, Phys. Rev. B 62, 15390 (2000).

[20] We measured a sample with $d_{1}=131 \mathrm{~nm}$. In this case, we did not observe clear evidence for the formation of electron puddles.

[21] Y. Hirayama, K. Muraki, and T. Saku, Appl. Phys. Lett. 72, 1745 (1998). 